\title{
BMJ Open New Onset of DiabetEs in aSsociation with pancreatic ductal adenocarcinoma (NODES Trial): protocol of a prospective, multicentre observational trial
}

\author{
Dóra Illés i , ${ }^{1}$ Emese Ivány, ${ }_{1}^{1}$ Gábor Holzinger, ${ }^{1}$ Klára Kosár, ${ }^{1}$ M Gordian Adam, ${ }^{2}$ \\ Beate Kamlage, ${ }^{2}$ Gábor Zsóri, ${ }^{1}$ Máté Tajti, ${ }^{1}$ Márk M Svébis, ${ }^{3}$ Viktor Horváth, ${ }^{3}$ \\ Ilona Oláh, ${ }^{4}$ Katalin Márta, ${ }^{5,6}$ Szilárd Váncsa, ${ }^{5,6}$ Noémi Zádori, ${ }^{7}$ Andrea Szentesi, ${ }^{7,8}$ \\ Bálint Czakó, ${ }^{9}$ Péter Hegyi (1) , ${ }^{7}$ László Czakó (1) ${ }^{10}$
}

To cite: Illés $D$, Ivány $E$, Holzinger G, et al. New Onset of DiabetEs in aSsociation with pancreatic ductal adenocarcinoma (NODES Trial): protocol of a prospective, multicentre observational trial. BMJ Open 2020;10:e037267. doi:10.1136/ bmjopen-2020-037267

- Prepublication history for this paper is available online. To view these files, please visit the journal online (http://dx.doi. org/10.1136/bmjopen-2020037267).

Received 29 January 2020 Revised 05 September 2020 Accepted 22 September 2020

Check for updates

(C) Author(s) (or their employer(s)) 2020. Re-use permitted under CC BY-NC. No commercial re-use. See rights and permissions. Published by BMJ.

For numbered affiliations see end of article.

Correspondence to

Dr László Czakó;

czako.laszlo@med.u-szeged.hu

\section{ABSTRACT}

Introduction Pancreatic ductal adenocarcinoma (PDAC) has a dismal prognosis with an overall 5-year survival of approximately $8 \%$. The success in reducing the mortality rate of PDAC is related to the discovery of new therapeutic agents, and to a significant extent to the development of early detection and prevention programmes. Patients with new-onset diabetes mellitus (DM) represent a high-risk group for PDAC as they have an eightfold higher risk of PDAC than the general population. The proposed screening programme may allow the detection of PDAC in the early, operable stage. Diagnosing more patients in the curable stage might decrease the morbidity and mortality rates of PDAC and additionally reduce the burden of the healthcare. Methods and analysis This is a prospective, multicentre observational cohort study. Patients $\geq 60$ years old diagnosed with new-onset ( $\leq 6$ months) diabetes will be included. Exclusion criteria are (1) Continuous alcohol abuse; (2) Chronic pancreatitis; (3) Previous pancreas operation/ pancreatectomy; (4) Pregnancy; (5) Present malignant disease and (6) Type 1 DM. Follow-up visits are scheduled every 6 months for up to 36 months. Data collection is based on questionnaires. Clinical symptoms, body weight and fasting blood will be collected at each, carbohydrate antigen 19-9 and blood to biobank at every second visit. The blood samples will be processed to plasma and analysed with mass spectrometry (MS)-based metabolomics. The metabolomic data will be used for biomarker validation for early detection of PDAC in the high-risk group patients with new-onset diabetes. Patients with worrisome features will undergo MRI or endoscopic ultrasound investigation, and surgical referral depending on the radiological findings. One of the secondary end points is the incidence of PDAC in patients with newly diagnosed DM.

Ethics and dissemination The study has been approved by the Scientific and Research Ethics Committee of the Hungarian Medical Research Council (41085-6/2019). We plan to disseminate the results to several members of the healthcare system includining medical doctors, dietitians, nurses, patients and so on. We plan to publish the results in a peer-reviewed high-quality journal for professionals. In addition, we also plan to publish it for lay readers in order to maximalise the dissemination and benefits of this trial.

\section{Strengths and limitations of this study}

- As patients are included prospectively, the study will yield a cohort to examine the metabolic changes that coincide with the occurrence of pancreatic ductal adenocarcinoma (PDAC) at a very early stage before it is diagnosed.

- The criteria for the diagnoses of diabetes and PDAC will be uniformly applied throughout the study period, moreover the diagnosis of pancreatic cancer will be confirmed with a high level of certainty in all subjects.

- Taking part in the screening is connected to a very low burden, as the blood collection is only minimally invasive.

- All patients will be monitored closely and frequently, which will increase the survival of all participants, especially the high-risk patients.

- It might be really difficult to include the required number of patients, considering that PDAC is a rare disease, and the elderly population has more comorbidities which makes our observations more difficult, and leads to a higher follow-up loss during the 36 months.

Trial registration number ClinicalTrials.gov NCT04164602

\section{INTRODUCTION}

Pancreatic ductal adenocarcinoma (PDAC) is a rare disease with a lifetime prevalence of $1.39 \%$, but its prevalence is continuously increasing. ${ }^{1-3}$ The prognosis is extremely poor: it has a 5-year survival rate of only $7 \%-8 \%,{ }^{4}$ and this rate has barely improved in the last 40 years. ${ }^{5}$ PDAC will be the second leading cause of cancer-related death by $2030 .^{6}$ The high mortality rate is a consequence of delayed diagnosis: in the absence of specific symptoms, PDAC is often diagnosed at an advanced stage. Surgery is the 
only curative treatment at this moment. Unfortunately, only $20 \%$ of the patients are eligible for curative resection at the time of the diagnosis because of the presence of metastases and locoregional infiltration. ${ }^{7}$ The success in reducing the mortality rate of PDAC is related to a significant extent to the development of early detection and prevention programmes. An effective screening programme is needed for the early diagnosis of PDAC in the asymptomatic stage to improve the prognosis. Due to the low lifetime prevalence, population-based screening is neither feasible nor cost-effective. It is recommended that subjects at high risk of PDAC should be screened. ${ }^{8}$

\section{PDAC and DM}

Patients with diabetes mellitus (DM) have an eightfold higher risk of developing PDAC within 2-3 years after the diagnosis of diabetes relative to the general population. ${ }^{9}$ In a meta-analysis which included 36 studies, individuals in whom DM had only recently been diagnosed ( $<4$ years) had a $50 \%$ increased risk of PDAC as compared with individuals who had diabetes for $>5$ years. ${ }^{10}$ Another meta-analysis of 35 cohort studies showed that DM was associated with an increased risk of PDAC (summary relative risks (RRs) $=1.94$; 95\% CI 1.66 to 2.27 ). Interestingly, the risk decreased with the duration of diabetes (5.38 for $<1$ year, 1.95 for $1-4$ years, 1.49 for $5-9$ years, 1.47 for $\geq 10$ years), thus providing evidence that diabetes in patients with PDAC is caused by the cancer itself. ${ }^{11}$ In these cases, patients are actually suffering from diabetes type 3c $(\mathrm{T} 3 \mathrm{cDM})$. Diabetes is already prevalent in small PDACs, ${ }^{12}$ and what is more important, is that diabetes occurs before the tumour is radiologically detectable. ${ }^{13}$ A populationbased study found that approximately $1 \%$ of patients with new-onset diabetes at age 50 years or older will be diagnosed with PDAC within 3 years of first meeting the criteria for diabetes, and $56 \%$ of these within 6 months of meeting the criteria for diabetes. ${ }^{9}$ Recognition of newonset diabetes as an early manifestation of PDAC could lead to diagnosis of asymptomatic, early stage PDAC. ${ }^{14}$ In our recent prospective study, the prevalence of PDAC in patients with new-onset diabetes was significantly higher than in the general population (the value of the standardised incidence ratio for PDAC in patients with newonset type 2 diabetes was 198.6 (95\% CI 6.25 to 46.9)); therefore, screening seems to be beneficial for detecting PDAC in this patient population. ${ }^{15}$ Weight loss in patients with pancreatic carcinoma-associated DM often precedes the onset of diabetes, while new-onset primary type $2 \mathrm{DM}$ is typically associated with weight gain. ${ }^{16}$ The paradoxical development of diabetes in the face of ongoing weight loss may be an important clue to diagnose PDAC in patients with new onset of diabetes.

\section{Screening modalities}

The carbohydrate antigen 19-9 (CA19-9) is currently the only blood-based biomarker in clinical use for PDAC. The sensitivity of this marker for PDAC is $75 \%$, the specificity is $90 \%$, the positive predictive value
(PPV) is $69 \%$ and the negative predictive value (NPV) is $90 \% .{ }^{17}$ These values fall below the required characteristics of a reliable screening test; ${ }^{10} 18$ therefore, serum CA19-9 measurement is not suitable for screening for PDAC. Imaging modalities represent the gold standard for diagnosing PDAC. The first choice is transabdominal ultrasonography, however, its sensitivity in PDAC diagnosis is only $50 \%-70 \%$. Its accuracy is low in tumours $<1 \mathrm{~cm}$, which are usually operable and negatively influenced by obesity and meteorism. ${ }^{19}$ CT has a better accuracy in diagnosing PDAC; however, the low prevalence of PDAC and radiation exposure associated with the modality prevents it from being used as a screening test. The odds for a correct diagnosis are also high employing endoscopic ultrasound (EUS) or endoscopic retrograde cholangiopancreatography, but again the low prevalence of PDAC in combination with the burden of the endoscopic intervention to the patient preclude the application of these diagnostic methods for screening. Furthermore, it is not economically feasible to employ CT or endoscopic imaging for screening as these methods are associated with high costs to the healthcare system.

The success of the strategy of using new-onset diabetes as a screening tool to identify subjects with a high likelihood of having asymptomatic PDAC will depend on our ability to differentiate PDAC-associated diabetes from the more common type 2 diabetes. PDAC-induced diabetes is thought to be a paraneoplastic phenomenon involving the release of products from the tumour rather than a result of the destruction of the pancreas due to malignant infiltration. ${ }^{2021}$ Data on incidence of PDAC in newonset DM is rare; numbers of $0.25 \%,{ }^{22} 0.85 \%{ }^{9}$ and $3.6 \%{ }^{23}$ have been reported. Therefore, to enable a diagnostic follow-up of new onset of diabetes, a further enrichment of this group is needed, ${ }^{142425}$ for example, elderly subjects (age is an independent risk factor for PDAC), weight $\operatorname{loss}^{26}$ or smoking.

A biomarker panel consisting of nine metabolites plus the established protein CA19-9 was recently identified by Mayerle et al with $89.9 \%$ sensitivity, $91.3 \%$ specificity and $99.8 \%$ NPV for differentiating PDAC from chronic pancreatitis. ${ }^{27}$ Employing the same methods, a biomarker panel for differential diagnosis between PDAC and noncancer-related diabetes was identified. The metabolite signature needs validation in an independent test cohort, which will be enabled with the present study. Provided the biomarker is validated, the panel could be effective for screening of the high-risk group patients diagnosed with new-onset DM.

\section{Aims of the project}

1. Estimate the incidence of PDAC in patients with newonset diabetes.

2. Diagnose PDAC in an early operable stage.

3. Validate a biomarker that distinguishes patients with PDAC-caused T3cDM from patients with type-2 diabetes mellitus (T2DM). 
Table 1 Diagnostic criteria of diabetes mellitus

\begin{tabular}{lll}
\hline Parameter & Value and unit & Description \\
\hline Fasting plasma glucose & $\begin{array}{l}\geq 126 \mathrm{mg} / \mathrm{dL} \\
(7.0 \mathrm{mmol} / \mathrm{L})\end{array}$ & Fasting is defined as no caloric intake for at least 8 hours. \\
2- hour plasma glucose & $\geq 200 \mathrm{mg} / \mathrm{dL}(11.1 \mathrm{mmol} / \mathrm{L})$ & $\begin{array}{l}\text { Oral glucose tolerance test. The test should be performed as } \\
\text { described by the WHO, using a glucose load containing the } \\
\text { equivalent of } 75 \mathrm{~g} \text { anhydrous glucose dissolved in water. }\end{array}$ \\
$\mathrm{HbA1c}$ & $\begin{array}{l}\geq .5 \% \\
(48 \mathrm{mmol} / \mathrm{mol})\end{array}$ & $\begin{array}{l}\text { The test should be performed in a laboratory using a method } \\
\text { that is National Glycohemoglobin Standardization Program } \\
\text { (NGSP) certified and standardised to the Diabetes Control and } \\
\text { Complications Trial (DCCT) assay. }\end{array}$ \\
\hline
\end{tabular}

\section{METHODS AND ANALYSIS Design}

This is a prospective, multicentre, observational cohort study aiming to validate a biomarker panel in the early stage of PDAC. The data collection is based on questionnaires, and blood samples will be drawn from all patients. The questionnaires (Form A at recruitment, Form B at every follow-up visit) will be filled by every included patient.

The inclusion criteria of this study are the following: (1) Patients over 60 years of age; (2) Diabetes diagnosed within 6 months (newly diagnosed)-diagnostical criteria are based on the Diabetes Control and Complications Trial (table 1). ${ }^{328}$ The participants signed written informed consent.

Exclusion criteria are as follows: (1) Continuous alcohol abuse; (2) Chronic pancreatitis; (3) Previous pancreas operation/pancreatectomy; (4) Pregnancy; (5) Present malignant disease; and (6) Type 1 DM. Patients with chronic pancreatitis were excluded because a metabolic signature differentiating between chronic pancreatitis and patients with PDAC has already been published $^{27}$ and is currently further evaluated by the META-PAC consortium, while the present study aims to differentiate between patients with PDAC-caused new-onset diabetes and new-onset diabetes due to other causes.

\section{Sample size}

Mayerle $e t a l^{27}$ found that the biomarker signature in question could distinguish patients with PDAC from those without with an $89.9 \%$ sensitivity (marginal error $8.9 \%$ ) and $81.3 \%$ specificity (marginal error 10.3\%). Chari et at concluded that elderly subjects with new-onset DM has eight times higher risk for pancreatic cancer than a person of similar age and sex without DM. In the light of the epidemiological data it is suggested that in Hungary the prevalence of PDAC is considerably higher than that compared with other countries, ${ }^{5}$ we assumed a $2 \%$ prevalence for PDAC. In reference to these data, sample size calculation suggests that 2661 patients will need to be enrolled in order to confirm or reject the hypothesis for the primary end point with a $10 \%$ dropout, $80 \%$ power and $95 \%$ significance level. The recruitment period is planned to last 36 months, and all included patients will be followed for 36 months.
Duration

The first recruiting centre will be initialized on 1 July 2019. Start of the patient recruitment: 31 January 2020. Planned completion of the study recruitment: 30 January 2023.

\section{Clinical data and clinical end points}

\section{Essential baseline clinical data}

Age, sex, body weight, body mass index, date of DM diagnosis, date of sampling, comorbidities, antidiabetic medication, clinical symptoms, histology and stage of PDAC.

\section{Primary clinical end points}

The sensitivity, specificity, PPV, NPV and accuracy of the biomarker test.

\section{Secondary end points}

(1) Mortality rate of PDAC in patients with new-onset diabetes; (2) The proportion of localised and resectable PDAC; (3) Change in body weight before visit 1 and during visits 2-6; (4) Change in fasting blood glucose and haemoglobin A1c (HbAlc) before visit 1 and during visits 2-6; (5) Antidiabetic medications and the risk of PDAC; (6) Presence of concomitant diseases; (7) Smoking and alcohol intake; (8) Incidence of PDAC in patients with new-onset diabetes; (9) Cost-benefit analysis.

\section{Study protocol}

Patients with diabetes will be recruited by our diabetologist and collaborating family physicians based on a recent ( $<6$ months) laboratory test (table 1$)$. Visit 0 is scheduled within 2 weeks from the referral (figure 1). Patients who meet study entry criteria and no exclusion, will be informed and offered to participate in the study, however signed informed consent will be necessary for inclusion. Clinical data, body weight and worrisome features (unintentional weight loss: $5 \%$ of body weight within 6 months without knowing the reason, ${ }^{29}$ abdominal pain/ discomfort, abnormal laboratory data, unstable glucose metabolism despite adequate diet and medical treatment and without intercurrent infection) will be recorded at visit 0 , and a fasting blood sample will be taken for assessment of laboratory data and metabolomics. C-peptide and glutamic acid decarboxylase antibodies will be determined to classify diabetes at visit 0 . Patients with type 1 


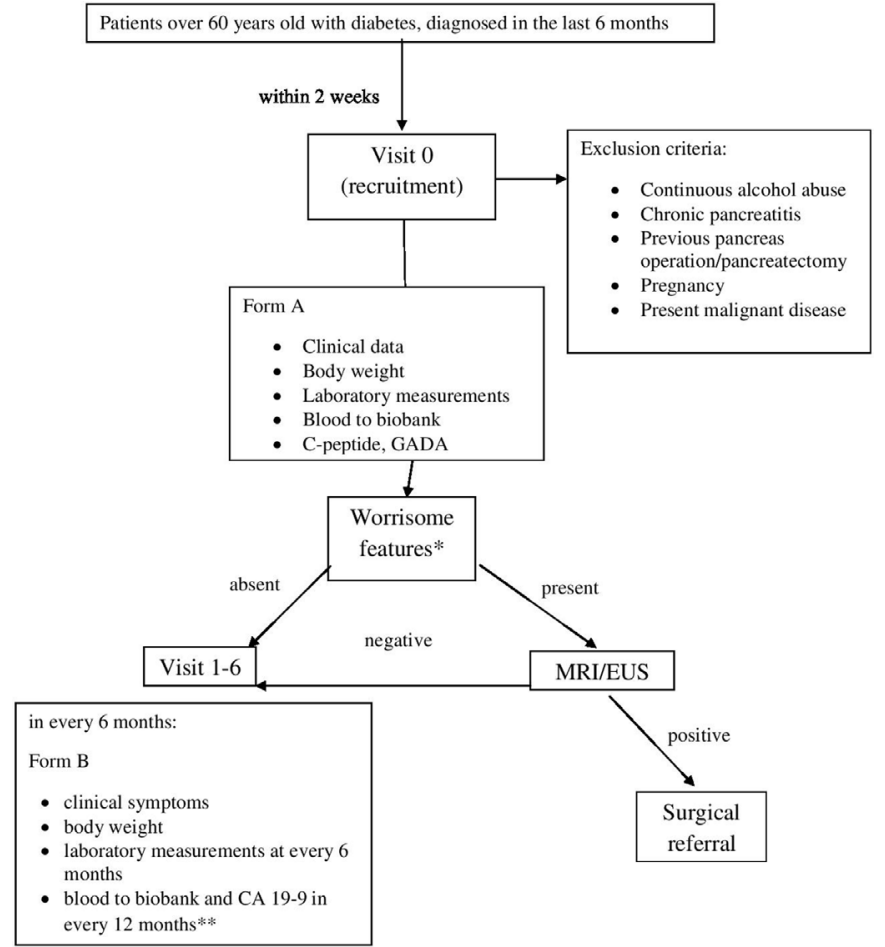

Figure 1 Flow chart of the study protocol. *Weight loss (except at visit 0), abdominal pain/discomfort, abnormal laboratory data, unstable glucose metabolism despite the adequate diet and medical treatment and without intercurrent infection (except at visit 0). ${ }^{\star \star}$ Fasted (overnight, at least 8 hours) patients' blood samples at room temperature will be drawn into an EDTA tube. Within 2 hours after blood draw samples will be at $19^{\circ} \mathrm{C}-21^{\circ} \mathrm{C}$. After centrifugation, the supernatant is carefully removed. After that, the plasma is transferred in $0.5 \mathrm{ml}$ aliquots to tubes and stored at $-80^{\circ} \mathrm{C}$, in a dedicated freezer ( $\leq 6$ hours from centrifuge to freezer). CA19-9, carbohydrate antigen 19-9; EUS, endoscopic ultrasound; GADA, glutamic acid decarboxylase antibodies.

DM will be excluded. If worrisome features are present at Visit 0, MRI or EUS is performed. Unambiguous PDAC lesions $(>1 \mathrm{~cm}$ or seen also by MRI) will be referred to surgery for resection. In case of ambiguous lesions in the pancreas, EUS-fine needle aspiration will be performed. Visits 1-5 are scheduled every 6 months. Clinical symptoms, body weight, laboratory data (fasting blood glucose, HbA1c, liver and renal function, lipids, blood count) will be collected at each visit. Blood to biobank and CA19-9 will be taken at every 12 months. The follow-up will be closed at 36 months.

\section{Biochemical methods}

After informed consent, fasted (overnight, at least 8 hours) patients' blood samples will be drawn into an EDTA tube. Blood tubes $(9 \mathrm{~mL})$ are centrifuged within 2 hours after blood draw using a swing-out rotor at $2000 \times g$ for $10 \mathrm{~min}$. The sample processing is done at room temperature and the centrifuge is temperature-controlled at $19^{\circ} \mathrm{C}-21^{\circ} \mathrm{C}$. After centrifugation, the supernatant is carefully removed, transferred to a fresh $9 \mathrm{~mL}$ tube and gently mixed in order to homogenise any gradient that might have been generated in the plasma supernatant. After that, the plasma is transferred in $0.5 \mathrm{~mL}$ aliquots to tubes (either Eppendorf Safe-Lock-Tubes $2 \mathrm{~mL}$ or Sarstedt Screw cap micro tubes $2 \mathrm{~mL}$ ) and stored at $-80^{\circ} \mathrm{C}$, in a dedicated freezer ( $\leq 6$ hours from centrifuge to freezer). Biomarkers will be determined comparing metabolite levels in plasma samples from patients diagnosed with PDAC and cancerfree patients with diabetes. ${ }^{26}$ CA19-9 determination is performed centralised at a certified clinical laboratory applying a cut-off of $37 \mathrm{U} / \mathrm{mL}$ as a classifier.

Cost of the biomarker test, quality-adjusted life years and incremental cost-effectiveness ratio will be determined.

\section{Metabolite profiling (MxP) \\ MxP global profiling}

Two types of mass spectrometry (MS) analyses are applied. GC-MS (gas chromatography-MS; Agilent 6890 GC coupled to an Agilent 5973 MS System, Agilent, Waldbronn, Germany) and liquid chromatography-MS/MS (LC-MS / MS; Agilent 1100 high performance LC (HPLC)System, Agilent, Waldbronn, Germany, coupled to an Applied Biosystems API4000 MS/MS-System, Applied Biosystems, Darmstadt, Germany) are used for a metabolite profiling approach. ${ }^{30}$ Fractionation and derivatisation of samples and detection technologies have been previously described. ${ }^{31-34}$ Proteins are removed from plasma samples $(60 \mu \mathrm{l})$ by precipitation. Subsequently, polar and non-polar fractions are separated for both GC-MS and LC-MS/MS analyses by adding water and a mixture of ethanol and dichloromethane. For GC-MS analyses, the non-polar fraction is treated with methanol under acidic conditions to yield the fatty acid methyl esters derived from free fatty acids and hydrolysed complex lipids. The polar and non-polar fractions are further derivatised with O-methyl-hydroxylamine hydrochloride $(20 \mathrm{mg} / \mathrm{mL}$ in pyridine) to convert oxo-groups to O-methyloximes and subsequently with a silylating agent (N-Methyl-N(trimethylsilyl) trifluoroacetamide) before GC-MS analysis. For LC-MS/MS analyses, both fractions are dried and subsequently reconstituted in appropriate solvent mixtures. HPLC is performed by gradient elution using methanol/water/formic acid on reversed phase separation columns.

\section{MxP lipids}

MxP lipids cover profiling of sphingolipids (ceramides, sphingomyelins and sphingobases). The metabolites are analysed in a semiquantitative approach (ie, relative to a pool). Total lipids are extracted from plasma by liquid/ liquid extraction using chloroform/methanol. The lipid extracts are subsequently fractionated by normal phase liquid LC into different lipid groups according to the references. ${ }^{3135}$ The fractions are analysed by LC-MS/MS using electrospray ionisation and atmospheric pressure chemical ionisation with detection of specific multiple reaction monitoring transitions for sphingomyelins and ceramides, respectively. 


\section{Data normalisation}

Details of data normalisation have been published. ${ }^{27}$ Metabolite profiling based on a semiquantitative analytical platform results in relative metabolite levels ('ratio') to a defined reference. To support this concept and to allow an alignment of different analytical batches, two different reference sample types are run in parallel throughout the whole process. First, a project pool is generated from aliquots of all samples and measured with four replicates within each analytical sequence that comprised 24 samples. For all semiquantitatively analysed metabolites, the results of each analyte from each sample are normalised against the median of the corresponding analyte in the pool reference samples within each analytical sequence to provide pool-normalised ratios. This process step compensates for interinstrumental and intrainstrumental variation, that is, variability that occurs when different analytical sequences are analysed by different devices. Second, to allow for an experiment-toexperiment alignment of semiquantitative data, MxPool (a large pool of a commercial human EDTA plasma suited for alignment of MxP studies) is analysed with 12 replicated samples, and the pool-normalised ratios are further normalised to the median of the MxPool samples, that is, ratios from this study are on the same level and therefore comparable with data from other studies normalised to other aliquots of the same MxPool. A rigorous quality control is performed on peak, analyte and sample levels and has been described previously. ${ }^{36}$

\section{Data collection and follow-up}

Data collection is based on questionnaires, and will be stored in a personalised electronic database (electronic case report form - eCRF). Form A: contains all antropometric parameters, routine clinical chemistry tests, fasting blood glucose and HbA1c. Follow-up visits will be scheduled by the patient registration system every 6 months. Blood will be taken for biomarker identification with metabolomics and CA19-9 determination at every 12 months. The total follow-up period is 3 years.

Pancreas adenocarcinoma will be diagnosed by histological examination.

\section{Data set analysis and normalisation}

Descriptive statistics-mean, median, SD, quartiles and relative frequency-relative risk (dichotomous variables), independent two-sample $\mathrm{T}$ test (continuous variable) in the case of normal distribution, furthermore MannWhitney $\mathrm{U}$ test in case of lack of normal distribution will be performed. Logistic regression will be applied for exploring predictive factors. Affiliated statistical analyses will be performed with an error probability of 0.05 (type-I error probability).

Prior to statistical analysis, $\log 10$ transformation of ratios is conducted so that the data distribution becomes approximately normal. SIMCA-P V.14.0 (Umetrics AB, Umea, Sweden), TIBCO Spotfire V.7.12.0 and R V.3.3.4 are used for data analyses and visualisations. Initially, an exploratory multivariate analysis (principal component analysis) is applied to $\log 10$-transformed ratios scaled to unit variance.

A simple linear model (ANOVA, package nlme) addressing additional clinical information and potentially confounding factors such as 'disease', 'age', 'body mass index', 'gender' and 'sample storage time' as fixed effects is fitted to the data. Significance level is set to $5 \%$. The multiple test problem for the number of metabolites is addressed by calculating the false discovery rate using the Benjamini and Hochberg method. ${ }^{37}$

To classify patients depending on their metabolic profiles a penalised logistic regression is fitted via Elastic Net Algorithm using the R package glmnet. ${ }^{37}$ Equal penalties are used for both the L1 and the L2 norms. Afterwards the cut-off established previously on the biomarker identification data set is applied on the test data without retraining, and the performance is measured in terms of area under the curve (AUC), sensitivity and specificity. Confidence levels for the AUC are calculated using the binormal model for the receiver operating characteristic curve. When the sensitivity is fixed at a particular value, PPV, NPV and the accuracy become monotone functions of the specificity; and CIs for these estimates are obtained by transformation of the CI for the specificity. CIs for sensitivity, specificity and accuracy are obtained for the cut-off prespecified in the training data by the method of Clopper and Pearson for the binomial distribution. For PPV and NPV the CIs will be obtained by the method of Gart and $\mathrm{Nam}^{38}$ for ratios of binomial parameters as implemented in the $\mathrm{R}$ package pairwise CI. ${ }^{39}$ When comparing the biomarker and CA19-9 on the test data, differences in sensitivity and specificity will be tested with McNemar's test.

\section{Centres}

The study will start with the following centres: University of Szeged, University of Pécs, University of Semmelweis, however, other centres are welcome to participate as an open label study. Completion of the letter of intent form will be mandatory for registering the participation of each institution.

\section{PATIENT AND PUBLIC INVOLVEMENT}

No patients are involved.

\section{ETHICS AND DISSEMINATION \\ Trial registration}

The trial has been registered at the ClinicalTrials.gov (NCT04164602).

\section{Ethical approval}

The study has been approved by the Scientific and Research Ethics Committee of the Hungarian Medical Research Council (41085-6/2019). Protocol version: V1.0 08.01.2019. 


\section{Publication policy}

Centres providing more than 50 patients can provide authors to the authorship list.

\section{Dissemination policy}

We plan to disseminate the results to several members of the healthcare system including medical doctors, dietitians, nurses, patients, and so on. We plan to publish the results in a peer-reviewed high-quality journal for professionals. In addition, we also plan to publish it for lay readers in order to maximalise the dissemination and benefits of this trial.

\section{DISCUSSION}

PDAC has a dismal prognosis, which is due to its late diagnosis. The success in reducing the mortality rate of PDAC is related to the development of early detection and prevention programmes. Age and DM are known as risk factors of PDAC. ${ }^{9-11} 1415$

The expected positive end point of this study is to validate a biomarker panel in elderly patients diagnosed with diabetes; whether it is suitable for early stage diagnosis of a mostly incurable, high-mortality cancer, when surgery is still possible and the cancer can be cured. PDAC-induced diabetes belongs to the group T3cDM and in parallel, T3cDM means the highest-risk group for PDAC. Unfortunately, it is still underdiagnosed in the clinical practicemaybe because its symptoms are very similar to T2DM's and its diagnosis is based on complex, expensive tests that are not routinely available. ${ }^{40}$ To diagnose patients with T3cDM based on these criteria would lead to enormous difficulties and it would not be a cost-effective screening method, which is unfavourable. While there are several pancreatic diseases that can cause T3cDM, this study focuses on the differences between PDAC-T3cDM and T2DM only. In that manner, this biomarker panel could be a diagnostic tool for the T3cDM subgroup PDAC-T3cDM. The test requires only one blood sample collection, which means that it is simple, repeatable, tolerable, minimally invasive, nearly painless, widely achievable and relatively cheap-it fulfils all the criteria set for a screening method. Identifying PDAC in an earlier (still resectable) stage through surveillance of high-risk patients would increase surgical resection rate, cure rates and survival by $30 \%-40 \%$. It would save lives, maintain better well-being among the population and would have an enormous financial benefit: the increasing number of successful surgical interventions leads to lower necessity of chemotherapy and palliative interventions (such as stent implantations or gastroenteroanastomosis operations); moreover it lowers the burden of the healthcare cost.

\section{Trial organisation, committees and boards}

The coordinator of the NODES Study is LC with the support of the Hungarian Pancreatic Study Group (HPSGcoordinating society, https://tm-centre.org/en/studygroups/hungarian-pancreatic-study-group/). HPSG has been running high-quality international, multicentre clinical trials since 2014 and has published the relevant guidelines for pancreatic diseases to improve patient care in pancreatology. ${ }^{41-49}$

The trial will be supported by the following committees: Steering Committee (SC): This committee will be led by $\mathrm{PH}$ (gastroenterologist and internal medicine specialist). DI and EI will be the members in Szeged (HU).

International Translational Advisory Board: This board will involve gastroenterologists. The International Translational Advisory Board will regularly monitor the progress of the trial and might give recommendations to the SC.

Data Monitoring Committee: The Data Monitoring Committee will handle all the data and ensure that the data in the eCRF is accurate, complete and legible. The Data Management Plan will describe the detailed data flow. The data manager will validate the data from completed eCRFs, according to a Data Cleaning Plan. Any missing, implausible or inconsistent recordings in the eCRFs will be referred back to the investigator using a data query form, and will be documented for each individual subject before clean file status is declared. All changes to eCRFs will be recorded.

\section{Author affiliations}

${ }^{1}$ First Department of Medicine, University of Szeged Faculty of Medicine, Szeged, Hungary

${ }^{2}$ Tegeler Weg 33, 10589, Metanomics Health GmbH, Berlin, Germany ${ }^{3}$ Department of Internal Medicine, Semmelweis University of Medicine, Budapest, Hungary

${ }^{4}$ Ilona Tóth Outpatient Clinic, Budapest, Hungary

${ }^{5}$ Institute for Translational Medicine, University of Pécs Medical School, Pécs, Hungary

${ }^{6}$ János Szentágothai Research Center, University of Pécs, Pécs, Hungary ${ }^{7}$ Institute for Translational Medicine, Pecsi Tudomanyegyetem Altalanos Orvostudomanyi Kar, Pecs, Hungary

${ }^{8}$ MTA-SZTE Translational Gastroenterology Research Group, Szegedi

Tudomanyegyetem, Szeged, Hungary

${ }^{9}$ Medical School, University of Szeged Faculty of Medicine, Szeged, Hungary

${ }^{10}$ First Department of Medicine, University of Szeged, Szeged, Hungary

Contributors The study was designed by DI, LC, BK. DI, LC, MGA drafted the manuscript. All authors edited, read and approved the final manuscript. Literature search, statistical calculation and figures preparation will be done by SV, NZ, AS, $\mathrm{BC}, \mathrm{KM}$. The Steering Committee will be led by PH. During the study DI, El, GH, KK, IO, GZ, MT, MMS, VH, LC are going to collect the patients.

Funding The study was supported by National Research, Development and Innovation Office grants (K128222 to LC) and by the ÚNKP-20-6 - New national excellence program of the Ministry for Innovation and Technology from the source of the National Research, Development and Innovation Fund. Centre costs (IT, biostatistics, trial organisation, etc) are covered by the University of Pécs Medical School, Momentum Grant of the Hungarian Academy of Sciences (LP201410/2014); and Economic Development and Innovation Operative Programme Grant and Highly Cited Publication Grant of the National Research, Development and Innovation Office (GINOP-2.3.2-15-2016-00048 Stay Alive, KH-125678 and EFOP 3.6.2-16-2017-00006 Live Longer).

Competing interests BK and AMG are employees of Metanomics Health GmbH, Germany.

Patient and public involvement Patients and/or the public were not involved in the design, or conduct, or reporting, or dissemination plans of this research.

Patient consent for publication Not required.

Provenance and peer review Not commissioned; externally peer reviewed.

Open access This is an open access article distributed in accordance with the Creative Commons Attribution Non Commercial (CC BY-NC 4.0) license, which 
permits others to distribute, remix, adapt, build upon this work non-commercially, and license their derivative works on different terms, provided the original work is properly cited, appropriate credit is given, any changes made indicated, and the use is non-commercial. See: http://creativecommons.org/licenses/by-nc/4.0/.

\section{ORCID iDs}

Dóra Illés http://orcid.org/0000-0003-3138-8039

Péter Hegyi http://orcid.org/0000-0002-7035-941X

László Czakó http://orcid.org/0000-0002-6331-0802

\section{REFERENCES}

1 National Cancer Institute. SEER cancer statistics review 1975-2006.

2 Hariharan D, Saied A, Kocher HM. Analysis of mortality rates for pancreatic cancer across the world. HPB 2008;10:58-62.

3 Malvezzi M, Bertuccio P, Levi F, et al. European cancer mortality predictions for the year 2013. Ann Oncol 2013;24:792-800.

4 Siegel RL, Miller KD, Jemal A. Cancer statistics, 2016. CA Cancer J Clin 2016;66:7-30.

5 Rawla P, Sunkara T, Gaduputi V. Epidemiology of pancreatic cancer: global trends, etiology and risk factors. World J Oncol 2019;10:10-27.

6 Rahib L, Smith BD, Aizenberg R, et al. Projecting cancer incidence and deaths to 2030: the unexpected burden of thyroid, liver, and pancreas cancers in the United States. Cancer Res 2014;74:2913-21.

7 Gillen S, Schuster T, Meyer Zum Büschenfelde C, et al. Preoperative/ neoadjuvant therapy in pancreatic cancer: a systematic review and meta-analysis of response and resection percentages. PLoS Med 2010;7:e1000267.

8 Brand RE, Lerch MM, Rubinstein WS, et al. Advances in counselling and surveillance of patients at risk for pancreatic cancer. Gut 2007;56:1460-9.

9 Chari ST, Leibson CL, Rabe KG, et al. Probability of pancreatic cancer following diabetes: a population-based study. Gastroenterology 2005;129:504-11.

10 Huxley R, Ansary-Moghaddam A, Berrington de González A, et al. Type-II diabetes and pancreatic cancer: a meta-analysis of 36 studies. Br J Cancer 2005;92:2076-83.

11 Ben Q, Xu M, Ning X, et al. Diabetes mellitus and risk of pancreatic cancer: a meta-analysis of cohort studies. Eur J Cancer 2011;47:1928-37.

12 Tsuchiya R, Noda T, Harada N, et al. Collective review of small carcinomas of the pancreas. Ann Surg 1986;203:77-81.

13 Pelaez-Luna M, Takahashi N, Fletcher JG, et al. Resectability of presymptomatic pancreatic cancer and its relationship to onset of diabetes: a retrospective review of CT scans and fasting glucose values prior to diagnosis. Am J Gastroenterol 2007;102:2157-63.

14 Pannala R, Basu A, Petersen GM, et al. New-onset diabetes: a potential clue to the early diagnosis of pancreatic cancer. Lancet Oncol 2009;10:88-95

15 Illés D, Terzin V, Holzinger G, et al. New-onset type 2 diabetes mellitus--A high-risk group suitable for the screening of pancreatic cancer? Pancreatology 2016;16:266-71.

16 Hart PA, Kamada P, Rabe KG, et al. Weight loss precedes cancerspecific symptoms in pancreatic cancer-associated diabetes mellitus. Pancreas 2011;40:768-72.

$17 \mathrm{Kim}$ J-E, Lee KT, Lee JK, et al. Clinical usefulness of carbohydrate antigen $19-9$ as a screening test for pancreatic cancer in an asymptomatic population. J Gastroenterol Hepatol 2004;19:182-6.

18 Maxim LD, Niebo R, Utell MJ. Screening tests: a review with examples. Inhal Toxicol 2014;26:811-28.

19 Rickes S, Unkrodt K, Neye H, et al. Differentiation of pancreatic tumours by conventional ultrasound, unenhanced and echoenhanced power Doppler sonography. Scand J Gastroenterol 2002;37:1313-20.

20 Pannala R, Leirness JB, Bamlet WR, et al. Prevalence and clinical profile of pancreatic cancer-associated diabetes mellitus. Gastroenterology 2008;134:981-7.

21 Aggarwal G, Ramachandran V, Javeed N, et al. Adrenomedullin is up-regulated in patients with pancreatic cancer and causes insulin resistance in $\beta$ cells and mice. Gastroenterology 2012;143:1510-7.

22 Munigala S, Singh A, Gelrud A, et al. Predictors for pancreatic cancer diagnosis following new-onset diabetes mellitus. Clin Trans/ Gastroenterol 2015;6:e118.

23 Choe JW, Kim JS, Kim HJ, et al. Value of early check-up of carbohydrate antigen 19-9 levels for pancreatic cancer screening in asymptomatic new-onset diabetic patients. Pancreas 2016;45:730-4
24 Muniraj T, Chari ST. Diabetes and pancreatic cancer. Minerva Gastroenterol Dietol 2012;58:331-45.

25 Chari ST, Kelly K, Hollingsworth MA, et al. Early detection of sporadic pancreatic cancer: summative review. Pancreas 2015;44:693-712.

26 Sah RP, Nagpal SJS, Mukhopadhyay D, et al. New insights into pancreatic cancer-induced paraneoplastic diabetes. Nat Rev Gastroenterol Hepatol 2013;10:423-33.

27 Mayerle J, Kalthoff H, Reszka R, et al. Metabolic biomarker signature to differentiate pancreatic ductal adenocarcinoma from chronic pancreatitis. Gut 2018;67:128-37.

28 American Diabetes Association. 2. Classification and Diagnosis of Diabetes: Standards of Medical Care in Diabetes-2018. Diabetes Care 2018;41:S13-27.

29 Rabinovitz M, Pitlik SD, Leifer M, et al. Unintentional weight loss. A retrospective analysis of 154 cases. Arch Intern Med 1986;146:186-7.

30 Jung K, Reszka R, Kamlage B, et al. Tissue metabolite profiling identifies differentiating and prognostic biomarkers for prostate carcinoma. Int J Cancer 2013;133:2914-24.

31 Christie WW. Rapid separation and quantification of lipid classes by high performance liquid chromatography and mass (light-scattering) detection. J Lipid Res 1985;26:507-12.

32 Mutch DM, Fuhrmann JC, Rein D, et al. Metabolite profiling identifies candidate markers reflecting the clinical adaptations associated with Roux-en-Y gastric bypass surgery. PLoS One 2009;4:e7905.

33 Roessner U, Wagner C, Kopka J, et al. Technical advance: simultaneous analysis of metabolites in potato tuber by gas chromatography-mass spectrometry. Plant J 2000;23:131-42.

34 van Ravenzwaay B, Cunha GC-P, Leibold E, et al. The use of metabolomics for the discovery of new biomarkers of effect. Toxicol Lett 2007;172:21-8.

35 Schmidt H, Schmidt R, Geisslinger G. LC-MS/MS-analysis of sphingosine-1-phosphate and related compounds in plasma samples. Prostaglandins Other Lipid Mediat 2006;81:162-70.

36 Meller S, Meyer H-A, Bethan B, et al. Integration of tissue metabolomics, transcriptomics and immunohistochemistry reveals ERG- and Gleason score-specific metabolomic alterations in prostate cancer. Oncotarget 2016;7:1421-38.

37 Benjamini Y, Hochberg Y. Controlling the false discovery rate: a practical and powerful approach to multiple testing. J R Stat Soc Series B 1995;57:289-300.

38 Gart JJ, Nam J. Approximate interval estimation of the ratio of binomial parameters: a review and corrections for skewness. Biometrics 1988:44:323-38.

39 Scherer R, Schaarschmidt F, Prescher S, et al. Simultaneous confidence intervals for comparing biodiversity indices estimated from overdispersed count data. Biom J 2013:55:246-63.

40 Ewald N, Bretzel RG. Diabetes mellitus secondary to pancreatic diseases (Type 3c)--are we neglecting an important disease? Eur $J$ Intern Med 2013;24:203-6.

41 Hritz I, Hegyi P. Early achievable severity (easy) index for simple and accurate expedite risk stratification in acute pancreatitis. $J$ Gastrointestin Liver Dis 2015;24:177-82.

42 Párniczky A, Mosztbacher D, Zsoldos F, et al. Analysis of pediatric pancreatitis (APPLE trial): Pre-study protocol of a multinational prospective clinical trial. Digestion 2016;93:105-10.

43 Zsoldos F, Párniczky A, Mosztbacher D, et al. Pain in the early phase of pediatric pancreatitis (PINEAPPLE trial): Pre-Study protocol of a multinational prospective clinical trial. Digestion 2016;93:121-6.

44 Dubravcsik Z, Madácsy L, Gyökeres T, et al. Preventive pancreatic stents in the management of acute biliary pancreatitis (PREPAST trial): pre-study protocol for a multicenter, prospective, randomized, interventional, controlled trial. Pancreatology 2015; $15: 115-23$

45 Márta K, Szabó AN, Pécsi D, et al. High versus low energy administration in the early phase of acute pancreatitis (GOULASH trial): protocol of a multicentre randomised double-blind clinical trial. BMJ Open 2017;7:e015874.

46 Dubravcsik Z, Farkas G, Hegyi P, et al. [Autoimmune pancreatitis. Evidence based management guidelines of the Hungarian Pancreatic Study Group]. Orv Hetil 2015;156:292-307.

47 Hritz I, Czakó L, Dubravcsik Z, et al. [Acute pancreatitis. Evidencebased practice guidelines, prepared by the Hungarian Pancreatic Study Group]. Orv Hetil 2015;156:244-61.

48 Párniczky A, Czakó L, Dubravcsik Z, et al. Pediatric pancreatitis. Evidence based management guidelines of the Hungarian Pancreatic Study Group. Orv Hetil 2015;156:308-25.

49 Takács T, Czakó L, Dubravcsik Z, et al. [Chronic pancreatitis. Evidence based management guidelines of the Hungarian Pancreatic Study Group]. Orv Hetil 2015;156:262-88. 